\title{
A generalization of the Goldbach-Vinogradov theorem
}

\author{
by
}

\author{
T. ZHAN (Jinan)
}

1. Introduction. The Goldbach-Vinogradov theorem states that every large odd integer is the sum of three primes. After Vinogradov proved this result in 1937, several generalizations of it were studied by a number of authors. One question is how small we may take $U=U(N)$ such that every large odd integer $N$ can be written in the form

(1.1) $\quad N=p_{1}+p_{2}+p_{3}, \quad p_{1} \leq U=U(N) \quad\left(2 \nmid N, p_{1}, p_{2}, p_{3}\right.$ are primes $)$.

The first non-trivial result concerning this problem was given by Pan [7], who proved in 1959 that $U$ may be taken as small as $N^{2 c /(2 c+1)+\varepsilon}$ if $\zeta\left(\frac{1}{2}+i t\right) \ll$ $(|t|+1)^{c+\varepsilon}$ for any $\varepsilon>0$. The classical result $c=1 / 6$ then gives $U=N^{1 / 4+\varepsilon}$. In the present paper some improvements on Pan's result will be given. Let

$$
R(N)=\sum_{\substack{p_{1}+p_{2}+p_{3}=N \\ p_{1} \leq U, p_{2} \leq y \\ N-y<p_{3} \leq N}} 1
$$

In Section 2 we prove

Theorem 1. Suppose that $0<\varepsilon<1 / 2, U=y^{1 / 6+\varepsilon}$ and $y=N^{7 / 12+\varepsilon}$. Then for $2 \nmid N$,

$$
R(N)=\sigma(N) U y L^{-1}(\log U)^{-1}(\log y)^{-1}\left(1+O\left(L^{-1}\right)\right),
$$

where

$$
\sigma(N)=\prod_{p \mid N}\left(1-\frac{1}{(p-1)^{2}}\right) \prod_{p \nmid N}\left(1+\frac{1}{(p-1)^{3}}\right)>\frac{1}{2} \quad \text { for } 2 \nmid N,
$$

$L=\log N$ and the $O$-constant depends on $\varepsilon$ only.

From Theorem 1 it follows that $U(N)=N^{7 / 72+\varepsilon}$ is permissible in (1.1).

Supported by the Alexander von Humboldt Foundation. 
The proof of Theorem 1 depends on the Hardy-Littlewood circle method and Selberg's inequality

$$
\int_{x}^{2 x}\left|\sum_{t<n \leq t+\Delta} \Lambda(n)-\Delta\right|^{2} d t \ll_{\varepsilon, A} \Delta^{2} x(\log x)^{-A},
$$

where $0<\varepsilon<5 / 6, A>0$ are any constants and $x^{1 / 6+\varepsilon} \leq \Delta \leq x$ (see [9], for example). In fact, what we actually need in the proof is a generalization of (1.3) stated in Lemma 1 in Section 2. The main idea of the proof is to make use of Gallagher's lemma [1] and (1.3) in estimating integrals on major arcs.

As a consequence of (1.3) we deduce that if $g(n)=n^{1 / 6+\varepsilon}$ then for all integers $n \leq x$ with at most $O\left(x(\log x)^{-A}\right)$ exceptions the interval $(n, n+$ $g(n))$ contains $\gg g(n)(\log n)^{-1}$ primes. A better result due to Harman [3] is known, namely, for $g(n)=n^{1 / 10+\varepsilon}$ the above statement is still true. Although it seems unlikely that one may get an improvement on Theorem 1 directly from Harman's result, we can prove the following Theorem 2 by combining the method of proof of Theorem 1 with Harman's sieve estimates used in proving his result. The idea of combining the circle method with the sieve method may be found in [5] or [6].

Theorem 2. Suppose that $0<\varepsilon<1 / 2, U=y^{1 / 10+\varepsilon}$ and $y=N^{7 / 12+\varepsilon}$. Then there exists an absolute constant $C_{1}>0$ such that

$$
R(N) \geq C_{1} U y L^{-3} .
$$

Theorem 2 implies that one may take $U(N)=N^{7 / 120+\varepsilon}$ in (1.1). The proof of Theorem 2 is given in Sections 3 and 4.

In what follows we shall use the notations introduced above. $c>0$ will denote a positive constant that may be different at each occurrence.

\section{Proof of Theorem 1}

Lemma 1. Suppose that $A>0, B>0$ and $0<\varepsilon<5 / 6$. Then for $(a, q)=1,1 \leq q \leq(\log x)^{B}$ and $x^{1 / 6+\varepsilon} \leq \Delta \leq x$ we have

$$
\int_{x}^{2 x}\left|\sum_{\substack{t<n \leq t+\Delta \\ n \equiv a(q)}} \Lambda(n)-\frac{\Delta}{\phi(q)}\right|^{2} d t \ll_{\varepsilon, c, A} \Delta^{2} x(\log x)^{-A} .
$$

This is a generalization of (1.3) and can be shown in essentially the same way as in [9]. 
For the sake of simplicity instead of $R(N)$ we consider $\widetilde{R}(N)$ defined by

$$
\widetilde{R}(N)=\sum_{\substack{N=n_{1}+n_{2}+n_{3} \\ n_{1} \leq U, n_{2} \leq y \\ N-y<n_{3} \leq N}} \Lambda\left(n_{1}\right) \Lambda\left(n_{2}\right) \Lambda\left(n_{3}\right)
$$

and prove

$$
\widetilde{R}(N)=\sigma(N) U y\left(1+O\left(L^{-1}\right)\right) .
$$

It is an easy matter to derive Theorem 1 from (2.2).

Applying the circle method, we obtain

$$
\begin{aligned}
\widetilde{R}(N)= & \int_{0}^{1} \sum_{n_{1} \leq U} \Lambda\left(n_{1}\right) e\left(n_{1} \alpha\right) \sum_{n_{2} \leq y} \Lambda\left(n_{2}\right) e\left(n_{2} \alpha\right) \\
& \times \sum_{N-y<n_{3} \leq N} \Lambda\left(n_{3}\right) e\left(n_{3} \alpha\right) e(-N \alpha) d \alpha \\
= & \left(\int_{m}+\int_{E}\right) \sum_{n_{1} \leq U} \Lambda\left(n_{1}\right) e\left(n_{1} \alpha\right) \sum_{n_{2} \leq y} \Lambda\left(n_{2}\right) e\left(n_{2} \alpha\right) \\
& \times \sum_{N-y<n_{3} \leq N} \Lambda\left(n_{3}\right) e\left(n_{3} \alpha\right) e(-N \alpha) d \alpha \\
= & \int_{m}+\int_{E}, \quad \text { say },
\end{aligned}
$$

where $m$ and $E$ are defined by

$$
\begin{gathered}
m=\bigcup_{\substack { q \leq Q \\
\begin{subarray}{c}{1 \leq a \leq q \\
(a, q)=1{ q \leq Q \\
\begin{subarray} { c } { 1 \leq a \leq q \\
( a , q ) = 1 } }\end{subarray}}^{\bigcup}\left[\frac{a}{q}-\frac{1}{q \tau}, \frac{a}{q}+\frac{1}{q \tau}\right], \quad Q=L^{c_{1}}, \tau=U L^{-c_{2}}, \\
E=[-1 / \tau, 1-1 / \tau] \backslash m .
\end{gathered}
$$

Denote by $I_{q, a}$ the interval $[a / q-1 /(q \tau), a / q+1 /(q \tau)]$. Taking $c_{1}=10$ and $c_{2}=12$, from the result of Vinogradov and the Siegel-Walfisz theorem (see [8], for example) we get

$$
\begin{gathered}
\sum_{n \leq U} \Lambda(n) e(n \alpha) \ll U L^{-2} \quad \text { if } \alpha \in E \\
\sum_{n \leq U} \Lambda(n) e(n \alpha)=\frac{\mu(q)}{\phi(q)} \sum_{n \leq U} e(n \lambda)+O\left(U \exp \left(-c L^{1 / 2}\right)\right) \\
\text { if } \alpha=a / q+\lambda \in m .
\end{gathered}
$$


Then it follows from (2.3) and the Cauchy-Schwarz inequality that

$$
\begin{aligned}
\int_{E} & \ll U L^{-2} \int_{0}^{1}\left|\sum_{n_{2} \leq y} \Lambda\left(n_{2}\right) e\left(n_{2} \alpha\right) \sum_{N-y<n_{3} \leq N} \Lambda\left(n_{3}\right) e\left(n_{3} \alpha\right)\right| d \alpha \\
& \ll y U L^{-1} .
\end{aligned}
$$

By (2.4) we obtain

$$
\begin{aligned}
(2.6) \int_{I_{q, a}}= & \frac{\mu(q)}{\phi(q)} \int_{-1 /(q \tau)}^{1 /(q \tau)} \sum_{n_{1} \leq U} e\left(n_{1} \lambda\right) \sum_{n_{2} \leq y} \Lambda\left(n_{2}\right) e\left(n_{2}(a / q+\lambda)\right) \\
& \times \sum_{N-y<n_{3} \leq N} \Lambda\left(n_{3}\right) e\left(n_{3}(a / q+\lambda)\right) e(-N(a / q+\lambda)) d \lambda \\
= & \frac{\mu^{2}(q)}{\phi^{2}(q)} \int_{-1 /(q \tau)}^{1 /(q \tau)} \sum_{n_{1} \leq U} e\left(n_{1} \lambda\right) \sum_{n_{2} \leq y} e\left(n_{2} \lambda\right) \\
& \times \sum_{N-y<n_{3} \leq N} \Lambda\left(n_{3}\right) e\left(n_{3}(a / q+\lambda)\right) e(-N(a / q+\lambda)) d \lambda \\
& +O\left(\frac{1}{\phi(q)} \int_{-1 / \tau}^{1 / \tau}\left|\sum_{n_{1} \leq U} e\left(n_{1} \lambda\right) \sum_{N-y<n_{3} \leq N} \Lambda\left(n_{3}\right) e\left(n_{3}(a / q+\lambda)\right)\right|\right. \\
& \left.\times\left|\sum_{n_{2} \leq y} \Lambda\left(n_{2}\right) e\left(n_{2}(a / q+\lambda)\right)-\frac{\mu(q)}{\phi(q)} \sum_{m \leq y} e(m \lambda)\right| d \lambda\right) \\
= & I_{1}+O\left(I_{2}\right), \quad \text { say. }
\end{aligned}
$$

By the Cauchy-Schwarz inequality and Gallagher's lemma [1] we have

$$
\begin{aligned}
\left|I_{2}\right|^{2} \ll & \phi^{-2}(q) U^{2} \int_{0}^{1}\left|\sum_{N-y<n \leq N} \Lambda(n) e(n(a / q+\lambda))\right|^{2} d \lambda \\
& \times \int_{-1 / \tau}^{1 / \tau}\left|\sum_{n \leq y} \Lambda(n) e(n(a / q+\lambda))-\frac{\mu(q)}{\phi(q)} \sum_{m \leq y} e(m \lambda)\right|^{2} d \lambda \\
\ll & \phi^{-2}(q) U^{2} y \tau^{-2} L \int_{1}^{y}\left|\sum_{t<n \leq t+\tau} \Lambda(n) e\left(n \frac{a}{q}\right)-\frac{\mu(q)}{\phi(q)} \tau\right|^{2} d t \\
& +\phi^{-2}(q) U^{2} y \tau L \\
\ll & U^{2} y \tau^{-2} L \max \int_{1}^{y}\left|\sum_{\substack{t<n \leq t+\tau \\
n \equiv l(q)}} \Lambda(n)-\frac{\tau}{\phi(q)}\right|^{2} d t+U^{2} y^{2-\varepsilon}
\end{aligned}
$$

where the "max" is taken over all $(l, q)=1$ and $q \leq Q$. Since $Q=L^{c_{1}}$, 
taking $A=4 c_{1}+2$ in Lemma 1 , we obtain

$$
I_{2} \ll Q^{-2} y U L^{-1} \text {. }
$$

We now turn to $I_{1}$. It is easy to check that

$I_{1}=\frac{\mu^{2}(q)}{\phi^{2}(q)} \int_{0}^{1} \sum_{n_{1} \leq U} e\left(n_{1} \lambda\right) \sum_{n_{2} \leq y} e\left(n_{2} \lambda\right)$

$$
\begin{aligned}
& \times \sum_{N-y<n_{3} \leq N} \Lambda\left(n_{3}\right) e\left(n_{3}(a / q+\lambda)\right) e(-N(a / q+\lambda)) d \lambda \\
& +O\left(\phi^{-2}(q) \int_{1 /(q \tau)}^{1 / 2} \lambda^{-1}\left|\sum_{n_{2} \leq y} e\left(n_{2} \lambda\right) \sum_{N-y<n_{3} \leq N} \Lambda\left(n_{3}\right) e\left(n_{3}(a / q+\lambda)\right)\right| d \lambda\right) \\
& =\frac{\mu^{2}(q)}{\phi^{2}(q)} \sum_{\substack{n_{1}+n_{2}+n_{3}=N \\
n_{1} \leq U, n_{2} \leq y \\
N-y<n_{3} \leq N}} \Lambda\left(n_{3}\right) e\left(\frac{a}{q}\left(n_{3}-N\right)\right)+O\left(\phi^{-1}(q) \tau y L\right) \\
& =\frac{\mu^{2}(q)}{\phi^{2}(q)} \sum_{N-y<n_{3} \leq N} \Lambda(n) e\left(\frac{a}{q}\left(n_{3}-N\right)\right) \sum_{\substack{n_{1}+n_{2}=N-n_{3} \\
n_{1} \leq U, n_{2} \leq y}} 1+O\left(\phi^{-1}(q) \tau y L\right) \\
& =\frac{\mu^{2}(q)}{\phi^{2}(q)} U \sum_{N-y<n \leq N} \Lambda(n) e\left(\frac{a}{q}(n-N)\right)+O\left(\phi^{-1}(q) \tau y L\right)+O\left(\phi^{-2}(q) U^{2}\right) \\
& =\frac{\mu^{2}(q)}{\phi^{2}(q)} U \sum_{\substack{l=1 \\
(l, q)=1}}^{q} e\left(\frac{a l}{q}\right) e\left(-\frac{a}{q} N\right) \sum_{\substack{N-y<n \leq N \\
n \equiv l(q)}} \Lambda(n)+O\left(\phi^{-1}(q) \tau y L\right) \\
& =\frac{\mu(q)}{\phi^{3}(q)} U y e\left(-\frac{a}{q} N\right)\left(1+O\left(L^{-1}\right)\right)+O\left(\phi^{-1}(q) \tau y L\right) \text {. }
\end{aligned}
$$

In the last step we used the Siegel-Walfisz theorem in short intervals, namely,

$$
\sum_{\substack{N-y<n \leq N \\ n \equiv l(q)}} \Lambda(n)=\frac{1}{\phi(q)} y+O\left(y \exp \left(-c L^{-1 / 2}\right)\right)
$$

holds for $(l, q)=1$ and $q \leq L^{B}$ ( $B>0$ is any constant).

From (2.6)-(2.8) it follows in a standard way that (see [8])

$$
\int_{m}=U y \sum_{q \leq Q} \frac{\mu(q)}{\phi^{3}(q)} e\left(-\frac{a}{q} N\right)+O\left(U y L^{-1}\right)=\sigma(N) U y\left(1+O\left(L^{-1}\right)\right) .
$$

(2.2) is thus proved. 
3. Preparation for the proof of Theorem 2. In this section we give an outline for the proof of Theorem 2 and some fundamental lemmas. Suppose that $\varepsilon>0$ is a constant sufficiently small, $N$ is an odd integer sufficiently large, $N^{7 / 12+\varepsilon} \leq y \leq N, Y \leq y \leq 2 Y$ and $y^{\varepsilon} \leq U \leq y^{1-\varepsilon}$. Consider the set

$$
\mathcal{A}=\mathcal{A}(N ; y, U)=\left\{f\left(p_{1}, p_{3}\right)=N-p_{1}-p_{3}: p_{1} \leq U, N-y<p_{3} \leq N\right\} .
$$

Since $-U \leq N-p_{1}-p_{3} \leq y$ for $N-p_{1}-p_{3} \in \mathcal{A}$, then

$$
R(N)=S\left(\mathcal{A}, y^{1 / 2}\right)+O\left(U^{2}\right) .
$$

From Buchstab's identity it follows that

$$
\begin{aligned}
S\left(\mathcal{A}, y^{1 / 2}\right)= & S\left(\mathcal{A}, z_{1}\right)-\sum_{\substack{z_{2} \leq p<y^{1 / 2} \\
z_{1} \leq p<z_{2}}} S\left(\mathcal{A}_{p}, z_{4}(p)\right)+\sum_{\substack{z_{3}(p) \leq q<p<y^{1 / 2} \\
p \geq z_{2}}} S\left(\mathcal{A}_{p q}, q\right) \\
& +\sum_{\substack{z_{4}(p) \leq q<p<z_{2} \\
p \geq z_{1}}} S\left(\mathcal{A}_{p q}, q\right) \\
= & \Sigma_{1}-\Sigma_{2}-\Sigma_{3}+\Sigma_{4}+\Sigma_{5}, \quad \text { say, }
\end{aligned}
$$

where the parameters $z_{i}(1 \leq i \leq 4)$ are as in Harman [3], namely,

$$
\begin{gathered}
z_{1}=Y^{26(1-3 \varepsilon) / 105}, \quad z_{2}=Y^{9 / 35}, \\
z_{3}(p)=\left(Y^{1-3 \varepsilon} p^{-1}\right)^{1 / 3}, \quad z_{4}(p)=\left(Y^{26 / 35-2 \varepsilon} p^{-1}\right)^{1 / 2} .
\end{gathered}
$$

Obviously, $\Sigma_{5} \geq 0$. If we replace $y^{1 / 2}$ by $(2 Y)^{1 / 2}$ in $\Sigma_{2}$ and $y^{1 / 2}$ by $Y^{1 / 2}$ in $\Sigma_{4}$, it follows that

$$
S\left(\mathcal{A}, y^{1 / 2}\right) \geq \Sigma_{1}-\Sigma_{2}-\Sigma_{3}+\Sigma_{4} .
$$

We shall give a lower bound for $\Sigma_{1}$ and an upper bound for $\Sigma_{2}$ and $\Sigma_{3}$ by Iwaniec's linear sieve (Lemma 2). To estimate the error terms arising in Lemma 1 we shall use Lemma 3 below which can be shown by the HardyLittlewood circle method as in the proof of Theorem 1. However, instead of Selberg's inequality here we have to use some sieve estimates already proved in Harman [3]. (We actually need some generalized forms of Harman's results.) Similar to estimating these error terms, an asymptotic formula for a subsum of $\Sigma_{4}$ can be given. In this case Lemma 4 will be used instead of Lemma 3. Then the theorem will follow if the right side of (3.2) is $\gg U y L^{-3}$.

Lemma 2 (Iwaniec [4]). Let $z \geq 2, D \geq z^{2}$ and $r(\mathcal{A}, d)=\left|\mathcal{A}_{d}\right|-W / d$. Then

$$
\begin{aligned}
& S(\mathcal{A}, z) \leq W V(z)\{F(s)+E\}+R^{+}, \\
& S(\mathcal{A}, z) \geq W V(z)\{f(s)-E\}-R^{-}
\end{aligned}
$$


where $s=(\log D) /(\log z)$ and $E=c \varepsilon+O\left((\log D)^{-1 / 3}\right)$. The remainder terms $R^{ \pm}$are of the form

$$
R^{ \pm}=\sum_{(D)} R_{(D)}^{ \pm}=\sum_{(D)} \sum_{v \leq D^{\varepsilon}} C_{(D)}^{ \pm}(v, \varepsilon) \sum_{D_{i} \leq p_{i} \leq D_{i}^{1+\varepsilon^{7}}}^{\prime} r\left(\mathcal{A}, v p_{1} \ldots p_{r}\right),
$$

where $(D)$ runs over all subsequences $D_{1} \geq \ldots \geq D_{r}$, including the empty subsequence, of the sequence $D^{\varepsilon^{2}\left(1+\varepsilon^{7}\right)^{n}}, n \geq 0$, for which

$$
D_{1} D_{2} \ldots D_{2 k} D_{2 k+1}^{3} \leq D \quad(0 \leq k \leq(r-1) / 2)
$$

in the case of $R^{+}$, and

$$
D_{1} D_{2} \ldots D_{2 k-1} D_{2 k}^{3} \leq D \quad(0 \leq k \leq r / 2)
$$

in the case of $R^{-}$. Moreover, $\sum^{\prime}$ indicates that $v$ and $p_{i}$ satisfy

$$
v\left|P\left(D^{\varepsilon^{2}}\right), \quad p_{i}\right| P(z) \quad(1 \leq i \leq r) .
$$

Finally, we also have $\left|C_{(D)}^{ \pm}(v, \varepsilon)\right| \leq 1$.

$F(s), f(s), P(z)$ and $V(z)$ are standard functions in sieve theory. For their properties see $[2]$.

In our case we take $W=\widetilde{U} \widetilde{y}$,

$$
\begin{aligned}
\widetilde{U} & =\sum_{p \leq U} 1=U(\log U)^{-1}\left(1+O\left(L^{-1}\right)\right), \\
\widetilde{y} & =\sum_{N-y<p \leq N} 1=y L^{-1}\left(1+O\left(L^{-1}\right)\right)
\end{aligned}
$$

and have

$$
V(z)=\prod_{p<z}\left(1-p^{-1}\right)=e^{-\gamma}(\log z)^{-1}\left(1+O\left((\log z)^{-1}\right)\right)
$$

where $\gamma$ is the Euler constant.

In Lemmas 3 and 4 we suppose that $c(n) \ll \tau^{c}(n)$ for $n \leq D, c(n)=0$ otherwise, and $T_{0}=\sum_{n \leq D} n^{-1} c(n) \gg 1$. Obviously, $T_{0} \ll L^{c}$ for $D \ll N$.

Lemma 3. Suppose that for any $A>0, B>0,0<\varepsilon \leq 9 / 10$ and $x^{1 / 10+\varepsilon} \leq \Delta \leq x$,

$$
\int_{x}^{2 x}\left|\sum_{\substack{t<n k \leq t+\Delta \\ n k \equiv a(q)}} c(n)-\frac{\Delta}{\phi(q)} T_{0}\right|^{2} d t \ll_{\varepsilon, A, B} \Delta^{2} x(\log x)^{-A}
$$

holds for $(a, q)=1$ and $q \leq(\log x)^{B}$. Then

$$
R_{1}(N)=\sum_{\substack{n k+p_{1}+p_{3}=N \\ p_{1} \leq U, n k \leq y \\ N-y<p_{3} \leq N}} c(n)=\sigma(N) U y T_{0}(\log U)^{-1} L^{-1}\left(1+O\left(L^{-1}\right)\right) .
$$


Pr o of. Similarly to the proof of Theorem 1 it suffices to show that

$$
\widetilde{R}_{1}(N)=\sum_{\substack{n k+n_{1}+n_{3}=N \\ n_{1} \leq U, n k \leq y \\ N-y<n_{3} \leq N}} \Lambda\left(n_{1}\right) \Lambda\left(n_{3}\right) c(n)=\sigma(N) U y T_{0}\left(1+O\left(L^{-1}\right)\right) .
$$

Suppose that $Q, \tau$, the major $\operatorname{arcs} m$ and the minor arcs $E$ are defined as in Section 2. Take $c_{1}$ and $c_{2}$ sufficiently large. In the same way as in Section 2 we get from Vinogradov's result

$$
\begin{aligned}
\widetilde{R}_{1}(N)= & \int_{0}^{1} \sum_{n_{1} \leq U} \Lambda\left(n_{1}\right) e\left(n_{1} \alpha\right) \sum_{n k \leq y} \Lambda(n k) e(n k \alpha) \\
& \times \sum_{N-y<n_{3} \leq N} \Lambda\left(n_{3}\right) e\left(n_{3} \alpha\right) e(-N \alpha) d \alpha \\
= & \int_{m}+\int_{E}=\int_{m}+O\left(y U T_{0} L^{-A}\right),
\end{aligned}
$$

and from (2.4) and (3.4),

$$
\begin{aligned}
\int_{I_{q, a}}= & \int_{-1 /(q \tau)}^{1 /(q \tau)} \sum_{n_{1} \leq U} \Lambda\left(n_{1}\right) e\left(n_{1}\left(\frac{a}{q}+\lambda\right)\right) \sum_{n k \leq y} c(n) e\left(n k\left(\frac{a}{q}+\lambda\right)\right) \\
& \times \sum_{N-y<n_{3} \leq N} \Lambda\left(n_{3}\right) e\left(n_{3}\left(\frac{a}{q}+\lambda\right)\right) e\left(-N\left(\frac{a}{q}+\lambda\right)\right) d \lambda \\
= & \frac{\mu^{2}(q)}{\phi^{2}(q)} \sum_{\substack{n_{1}+n k+n_{3}=N \\
n_{1} \leq U, n k \leq y \\
N-y<n_{3} \leq N}} \Lambda\left(n_{3}\right) c(n) e\left(\frac{a}{q}\left(n_{3}-N\right)\right)+O\left(\phi^{-1}(q) \tau y L\right) .
\end{aligned}
$$

To evaluate the integral $\int_{I_{q, a}}$ we first rewrite it as

$$
\begin{aligned}
\int_{I_{q, a}}= & \frac{\mu^{2}(q)}{\phi^{2}(q)} \sum_{\substack{l=1 \\
(l, q)=1}}^{q} e\left(\frac{a}{q}(l-N)\right) \sum_{\substack{N-y<n_{3} \leq N \\
n_{3} \equiv l(q)}} \Lambda\left(n_{3}\right) \\
& \times \sum_{\substack{n_{1}+n k=N-n_{3} \\
n_{1} \leq U, n k \leq y}} c(n)+O\left(\phi^{-1}(q) \tau y L\right) \\
= & \frac{\mu^{2}(q)}{\phi^{2}(q)} \sum_{\substack{l=1 \\
(l, q)=1}}^{q}\left(\frac{a}{q}(l-N)\right) \sum_{\substack{N-y<n_{3} \leq N-U \\
n_{3} \equiv l(q)}} \Lambda\left(n_{3}\right) \\
& \times \sum_{N-n_{3}-U \leq n k<N-n_{3}} c(n)+O\left(\phi^{-1}(q) \tau y L\right) .
\end{aligned}
$$


Making substitution $u=t+\Delta$ in $(3.5)(q=1)$, we obtain

$$
\int_{x}^{2 x}\left|\sum_{u-\Delta<n k \leq u} c(n)-\Delta T_{0}\right|^{2} d u \ll_{\varepsilon, A, B} \Delta^{2} x(\log x)^{-A}+\Delta^{3}(\log x)^{c} .
$$

Take $x=y$ and $\Delta=U$ in (3.7). It follows that

$$
\sum_{U<m \leq y}\left|\sum_{m-U<n k \leq m} c(n)-U T_{0}\right|^{2} \ll U^{2} y L^{-A} .
$$

Hence by the Cauchy-Schwarz inequality we have

$$
\sum_{U<m \leq y}\left|\sum_{m-U<n k \leq m} c(n)-U T_{0}\right| \ll U y L^{-A / 2} .
$$

(3.7), (3.9) and the Siegel-Walfisz theorem in short intervals (2.9) then yield

$$
\begin{aligned}
\int_{I_{q, a}}= & \frac{\mu^{2}(q)}{\phi^{2}(q)} U T_{0} \sum_{\substack{l=1 \\
(l, q)=1}}^{q} e\left(\frac{a}{q}(l-N)\right) \sum_{\substack{N-y<n_{3} \leq N-U \\
n_{3} \equiv l(q)}} \Lambda\left(n_{3}\right) \\
& +O\left(\phi^{-1}(q) \tau y L\right)+O\left(Q^{-2} L^{-1} y U\right) \\
= & \frac{\mu(q)}{\phi^{3}(q)} U y T_{0} e\left(-N \frac{a}{q}\right)+O\left(Q^{-2} L^{-1} y U\right)
\end{aligned}
$$

if $c_{1}, c_{2}$ are taken sufficiently large and $c_{2} \geq c_{1}+2$. Hence

$$
\int_{m}=U y T_{0} \sum_{q \leq Q} \frac{\mu(q)}{\phi^{3}(q)} e\left(-\frac{a}{q} N\right)+O\left(U y L^{-1}\right)=\sigma(N) U y T_{0}\left(1+O\left(L^{-1}\right)\right),
$$

and (3.6) follows.

Lemma 4. Suppose that for any $A>0, B>0,0<\varepsilon \leq 9 / 10$ and $x^{1 / 10+\varepsilon} \leq \Delta \leq x$,

$$
\int_{x}^{2 x}\left|\sum_{\substack{t<n k \leq t+\Delta \\ n k \equiv a(q)}} c(n) \Lambda(k)-\frac{\Delta}{\phi(q)} T_{0}\right|^{2} d t \ll_{\varepsilon, A, B} \Delta^{2} x(\log x)^{-A}
$$

holds for $(a, q)=1$ and $q \leq(\log x)^{B}$. Then

$$
R_{1}(N)=\sum_{\substack{n p+p_{1}+p_{3}=N \\ p_{1} \leq U, n k \leq y \\ N-y<p_{3} \leq N}} c(n)=\sigma(N) U y T_{0}(\log U)^{-1} L^{-1}\left(1+O\left(L^{-1}\right)\right) .
$$

This lemma can be shown in the same way as Lemma 3 .

4. Proof of Theorem 2. In this section we assume all the conditions of Theorem 2. For the sake of simplicity we only prove the theorem for $\varepsilon>0$ sufficiently small. In fact, this is the most important case, the other case 
can be treated in the same way. Furthermore, the constants $c_{i}(i=1,2, \ldots)$ in this section are independent of the $c_{i}$ 's in Section 3.

Estimation of $\Sigma_{1}$. Applying Lemma 2 to $\Sigma_{1}$ we obtain

$$
\Sigma_{1} \geq W V\left(z_{1}\right)\left(f\left(\log D / \log z_{1}\right)-c \varepsilon+O\left(L^{-1 / 3}\right)\right)-R^{-} .
$$

Take $D=y^{1-3 \varepsilon}$ as in [3]. From (3.3), (3.4) and the result in $[3, \S 5]$ it follows that

$$
\Sigma_{1} \geq c_{1} \widetilde{U} \widetilde{y}(\log y)^{-1}\left(1-c \varepsilon+O\left(L^{-1 / 3}\right)\right)-R^{-},
$$

where $c_{1}=2 \log (79 / 26)$. Writing $R^{-}$in the form

$$
R^{-}=\sum_{(D)} R_{(D)}^{-}=\sum_{(D)} \sum_{n \leq D} c(n) r(\mathcal{A}, n)
$$

it follows easily that $c(n) \ll \tau^{c}(n)$ and

$$
R_{(D)}^{-}=\sum_{\begin{array}{c}
p_{1}+n k+p_{3}=N \\
N-y<p_{3} \leq N \\
p_{1} \leq U, n k \leq y, n \leq D
\end{array}} c(n)-T_{0} W .
$$

Now,

$$
\sum_{\substack{t<n l \leq t+k \\ n \leq D}} c(n)-T_{0} U
$$

is actually the error term arising from the sieve estimate in [3] and it was already proved there that

$$
\int_{y / 2}^{y}\left|\sum_{\substack{t<n k \leq t+U \\ n \leq D}} c(n)-T_{0} U\right|^{2} d t \ll U^{2} y^{1-\delta} .
$$

By essentially the same method we can show that

$$
\int_{y / 2}^{y}\left|\sum_{\substack{t<n k \leq t+U \\ n \leq D, n \bar{k} \equiv a(q)}} c(n)-\frac{U}{\phi(q)} T_{0}\right|^{2} d t \ll_{\varepsilon, A, B} U^{2} y(\log x)^{-A} .
$$

Hence from Lemma 3 and (4.1) we get $R_{(D)}^{-} \ll U y^{1-\delta}$, and so

$$
\Sigma_{1} \geq c_{1} \widetilde{U} \widetilde{y}(\log y)^{-1}\left(1-c \varepsilon+O\left(L^{-1 / 3}\right)\right) .
$$

Estimation of $\Sigma_{2}$. Start from

$$
\begin{aligned}
\Sigma_{2} & =\sum_{z_{2} \leq p<(2 Y)^{1 / 2}} S\left(\mathcal{A}_{p}, z_{3}(p)\right) \\
& \leq \sum_{(P)}(\log P)^{-1} \sum_{P \leq n \leq 2 P} \Lambda(n) S\left(\mathcal{A}_{n}, z_{3}(p)\right) .
\end{aligned}
$$


Applying Lemma 2 to $S\left(\mathcal{A}_{n}, z_{3}(p)\right)$ with $D=Y^{1-3 \varepsilon} P^{-1}$ we obtain

$$
\begin{aligned}
\Sigma_{2} \leq & \sum_{z_{2} \leq p<(2 Y)^{1 / 2}} S\left(\mathcal{A}_{p}, z_{3}(p)\right) \sum_{P \leq n \leq 2 P} \Lambda(n) n^{-1} W V\left(z_{3}(P)\right) \\
& \times S\left(\mathcal{A}_{n}, z_{3}(P)\right)\left(F\left(\log D / \log z_{3}(P)\right)+c \varepsilon+O\left(L^{-1 / 3}\right)\right) \\
& +\sum_{(P)}(\log P)^{-1} \sum_{P \leq n \leq 2 P} \Lambda(n) R_{n}^{+} \\
= & \Sigma_{2}^{(1)}+\Sigma_{2}^{(2)}, \quad \text { say. }
\end{aligned}
$$

From the discussion in $[3, \S 5]$ we know that the main term

$$
\Sigma_{2}^{(1)} \leq c_{2} \widetilde{U} \widetilde{y}(\log y)^{-1}\left(1+c \varepsilon+O\left(L^{-1 / 3}\right)\right),
$$

where $c_{2}=2 \log (26 / 9)$. The error term may be written as

$$
\Sigma_{2}^{(2)}=\sum_{(P)}(\log P)^{-1} \sum_{(D)} R_{(D)}^{+}(P)
$$

with

$$
R_{(D)}^{+}(P)=\sum_{n \leq D} c(n) r(\mathcal{A}, n)=\sum_{\substack{p_{1}+n k+p_{3}=N \\ N-y<p_{3} \leq N \\ p_{1} \leq U, n k \leq y, n \leq Y^{1-3 \varepsilon}}} c(n)-T_{0} W .
$$

It is easy to see that

$$
\sum_{\substack{t<n k \leq t+U \\ n \leq Y^{1-3 \varepsilon}}} c(n)-T_{0} U
$$

is actually the error term of

$$
\sum_{P \leq n \leq 2 P} \Lambda(n) S\left(\mathcal{A}_{n}, z_{3}(p)\right)
$$

in [3]. Hence it follows in the same way as in the estimation of $\Sigma_{1}$ that

$$
\Sigma_{2}^{(2)} \ll U y^{1-\delta} \text {. }
$$

(4.2) and (4.3) yield

$$
\Sigma_{2} \leq c_{2} \widetilde{U} \widetilde{y}(\log y)^{-1}\left(1+c \varepsilon+O\left(L^{-1 / 3}\right)\right) .
$$

Estimation of $\Sigma_{3}$. Similarly to the treatment of $\Sigma_{2}$, it follows from Lemma 1, Lemma 3 and the corresponding estimates in $[3, \S 5]$ that

$$
\Sigma_{3} \leq c_{3} \widetilde{U} \widetilde{y}(\log y)^{-1}\left(1+c \varepsilon+O\left(L^{-1 / 3}\right)\right),
$$

where

$$
c_{3}=2 \log (2133 / 2028)(1+1 / 17-2 \log (35 / 34)) .
$$


Estimation of $\Sigma_{4}$. Again, similarly to the estimation of $\Sigma_{4}$ in [3] we can give an asymptotic formula for a subsum $\Sigma_{4}^{\prime}$ of $\Sigma_{4}$ (in the subsum $p$ and $q$ are taken over the same range as in [3]). We can apply Lemma 4 directly to $\Sigma_{4}^{\prime}$ since from $[3, \S 5]$ we know that the condition of Lemma 4 is fulfilled. Hence

$$
\Sigma_{4} \geq c_{4} \widetilde{U} \widetilde{y}(\log y)^{-1}\left(1-c \varepsilon+O\left(L^{-1}\right)\right),
$$

where $c_{4}=0.14$.

Since $c_{1}-c_{2}-c_{3}+c_{4}>0$, Theorem 2 is proved.

Acknowledgements. This paper was written while the author was visiting the Mathematical Institute of Freiburg University as a Research Fellow of the Humboldt Foundation. He would like to thank the Humboldt Foundation for its financial support, and Professor Pan Chengdong and Professor D. Wolke for careful reading of the manuscript and helpful discussions.

\section{References}

[1] P. X. Gallagher, A large sieve density estimate near $\sigma=1$, Invent. Math. 11 (1970), 329-339.

[2] H. Halberstam and H.-E. Richert, Sieve Method, Academic Press, 1974.

[3] G. Harman, Primes in short intervals, Math. Z. 180 (1982), 335-348.

[4] H. Iwaniec, A new form of the error term in the linear sieve, Acta Arith. 37 (1980), 307-320

[5] C. Jia, Three primes theorem in short intervals, to appear.

[6] H. Mikawa, On the exceptional set in Goldbach problem, to appear.

[7] Chengdong Pan, Some new results in additive number theory, Acta Math. Sinica 9 (1959), 315-329.

[8] Chengdong Pan and Chengbiao Pan, Goldbach Conjecture, Science Press, Peking, 1981.

[9] B. Saffari and R. C. Vaughan, On the fractional parts of $x / n$ and related sequences II, Ann. Inst. Fourier (Grenoble) 27 (1977), 1-30.

MATHEMATICAL INSTITUTE

SHANDONG UNIVERSITY

JINAN, SHANDONG 250100

P.R. CHINA 